\title{
Transverse impedance of axially symmetric tapered structures
}

\author{
B. Podobedov and S. Krinsky \\ Brookhaven National Laboratory, Upton, New York 11973, USA
}

(Received 4 April 2006; published 24 May 2006)

\begin{abstract}
We determine the low frequency transverse impedance of axially symmetric tapered structures. Higherorder perturbation theory is used to improve previous estimates due to Yokoya and Stupakov. For linear tapers, accurate numerical results are obtained using the ABCI electromagnetic simulation code. Based upon insight gained from the perturbation calculations, we introduce a simple parametrization that provides an excellent fit to all of our $\mathrm{ABCI}$ data-including cases with gradual and steep tapers as well as large and small change in cross section.
\end{abstract}

DOI: $10.1103 /$ PhysRevSTAB.9.054401

PACS numbers: $41.20 . \mathrm{Jb}, 41.60 .-\mathrm{m}$

\section{INTRODUCTION}

We consider the transverse impedance of axially symmetric tapered structures. Our chief interest is in situations where the change in radius is large and the corresponding transverse impedance needs to be minimized by having a tapered transition. This is commonly encountered in accelerator structures, such as transitions to radio-frequency cavities, small gap undulator chambers, beam collimators, etc. The geometric impedance at zero frequency is purely inductive [1]. When the transition is gradual, the low frequency transverse impedance [2,3] remains approximately at its zero-frequency value over a broad frequency range. In many cases of practical interest, this region of constant impedance may extend far enough in frequency to determine the kick factor and hence describe many transverse single bunch collective effects. Since space in an accelerator structure is precious, it is important to know how much tapering is required to avoid harmful impedance-related effects.

We have taken a two-pronged approach to solving this problem: analytical and numerical. Our analytic work is based upon a perturbation expansion for the transverse impedance at zero frequency which we derive by extending the method introduced by Stupakov [3]. The lowest order term, which was first derived by Yokoya [2], is accurate for very gradual and smooth tapers. Including higher-order terms allows us to treat steeper tapers and nonsmooth boundaries. Our numerical work has been carried out using the ABCI code [4]. Based upon the insight we gained from the analytic calculations, we have succeeded in finding a very simple parametrization that provides an excellent fit to all our numerical data. The goal of our work has been to obtain useful results for axially symmetric accelerator structures as well as to build up a theoretical foundation for future analyses of structures without axial symmetry, e.g., those with elliptical and flat cross sections [5].

Let us start by reviewing Yokoya's [2] result for the low frequency transverse impedance of an axially symmetric tapered transition,

$$
Z_{\perp}(k) \cong \frac{-i Z_{0}}{2 \pi} \int_{-\infty}^{\infty} d z \frac{r^{\prime}(z)^{2}}{r(z)^{2}},
$$

where $k$ is the wave number of the perturbing field, $Z_{0}$ is the free space impedance, $r(z)$ is the radius of the tapered chamber, and the prime denotes derivative with respect to the axial coordinate $z$. Criteria for the validity of Eq. (1.1) have been discussed by Yokoya [2] and Stupakov [3]. Based upon their discussion and our present work, we believe a useful criterion can be stated as follows: If the variation of the radius takes place over a characteristic distance $L$, then Eq. (1.1) holds under the conditions $r_{a v} \ll$ $L$ and $k r_{a v}^{2} \ll L$, where $r_{a v}$ is the average radius. In this paper, we derive an asymptotic expansion for the impedance at zero frequency in powers of $r_{a v} / L$.

The numerical calculations we have carried out using $\mathrm{ABCI}$ are sufficient to determine the low frequency transverse impedance for a linear taper of the form

$$
r(z)=\left\{\begin{array}{cc}
r_{\min }=r_{a v}(1-\varepsilon) & (z \leq-L) \\
r_{a v}\left(1+\frac{\varepsilon z}{L}\right) & (-L<z<L) \\
r_{\max }=r_{a v}(1+\varepsilon) & (L \leq z)
\end{array}\right\} .
$$

Since the first derivative $r^{\prime}(z)$ is discontinuous at $z= \pm L$, the analytic treatment of this case requires special consideration. Carefully applying perturbation theory to this singular case, we have shown that for $r_{a v} / L$ not too large

$$
Z_{\perp}(0) \cong-\frac{i Z_{0} \varepsilon^{2}}{2 \pi L} \frac{2}{1-\varepsilon^{2}}\left(1-0.18 \frac{r_{a v}}{L}\right) .
$$

Expressing this result in terms of the angle $\theta$ characterizing the linear taper, defined by

$$
\tan \theta=\frac{\varepsilon r_{a v}}{L},
$$

one finds

$$
Z_{\perp}(0) \cong-\frac{i Z_{0} \varepsilon}{2 \pi r_{a v}} \frac{2 \tan \theta}{1-\varepsilon^{2}}\left(1-0.18 \frac{\tan \theta}{\varepsilon}\right) .
$$

When we compared Eq. (1.5) with the results of $\mathrm{ABCI}$ calculations, we noticed that the agreement could be ex- 
tended to larger angle by replacing $\tan \theta$ by the angle $\theta$ itself. That is, we found better agreement using

$$
Z_{\perp}(0) \cong-\frac{i Z_{0} \varepsilon}{2 \pi r_{a v}} \frac{2 \theta}{1-\varepsilon^{2}}\left(1-0.18 \frac{\theta}{\varepsilon}\right)
$$

An excellent fit (see Fig. 4 below) to all of our ABCI results was obtained by assuming the impedance of a single taper to be expressed in the form

$$
Z_{\perp}(0) \cong-\frac{i Z_{0} \varepsilon}{2 \pi r_{a v}} \frac{2 \theta}{1-\varepsilon^{2}} \frac{1+(a+b \varepsilon) \frac{\theta}{\varepsilon}}{1+(0.18+a+c \varepsilon) \frac{\theta}{\varepsilon}},
$$

where the parameters $a, b, c$ were determined by least squares optimization, yielding

$$
\begin{gathered}
a=2.94 \times 10^{-3}, \quad b=-3.13 \times 10^{-3}, \\
c=1.75 \times 10^{-1} .
\end{gathered}
$$

Our paper is organized as follows: In Sec. II, we consider the derivation of the perturbation expansion for the transverse impedance at zero frequency and derive the result given in Eq. (1.3) for the case of a linear taper. In Sec. III, we discuss our ABCI calculations, and compare numerical results with the approximations given in Eqs. (1.1), (1.5), (1.6), and (1.7). Our conclusions are summarized in Sec. IV. In Appendix A, we show that for small $\varepsilon=\left(r_{\max }-r_{\min }\right) /\left(r_{\max }+r_{\min }\right)$, the results of our perturbation expansion agree with what is found using the boundary perturbation method [6,7]. In Appendix B, we consider in more depth some important details of the ABCI calculations. In particular, we explain how we carried out calculations for several grid sizes and obtained our final results by employing a linear extrapolation to vanishing grid spacing.

\section{PERTURBATION EXPANSION}

\section{A. Method}

Stupakov [3] has developed a perturbation theory to determine the transverse impedance at zero frequency of a circular tube with a smoothly varying radius. His approach is based on the solution of electrostatic and magnetostatic problems. Specifically, he determines the transverse impedance from the expression

$$
\operatorname{Im} Z_{\perp}(0)=\frac{Z_{0}}{4 \pi} \int_{-\infty}^{\infty} d z\left(\frac{\partial \Phi}{\partial x}-\frac{\partial \psi}{\partial y}\right)_{x=y=0},
$$

where $\Phi(\psi)$ is the electric (magnetic) potential due to a line electric (magnetic) dipole located on the $z$-axis. Electric (magnetic) dipole moment is oriented in $x(-y)$ direction and it has unit moment per unit of length. Potentials are found by perturbation theory, where for the zeroth order, Stupakov takes solutions for an untapered pipe. Carrying out this calculation to first order, he obtained the result (1.1) first found by Yokoya [2] by a different method. Here, we extend Stupakov's calculation to higher order.

In cylindrical coordinates the electric scalar potential is expanded in the form

$$
\Phi(r, \phi, z)=2 \cos \phi \sum_{n=0}^{\infty} f_{n}(r, z)
$$

where

$$
f_{0}(r, z)=\frac{1}{r}-\frac{r}{[r(z)]^{2}},
$$

and the other $f_{n}$ are determined by solving the recursion relations

$$
\frac{\partial^{2} f_{n}}{\partial r^{2}}+\frac{1}{r} \frac{\partial f_{n}}{\partial r}-\frac{f_{n}}{r^{2}}=-\frac{\partial^{2} f_{n-1}}{\partial z^{2}}
$$

subject to the boundary conditions

$$
f_{n}[r(z), z]=0 \quad \text { and } \quad f_{n}(0, z)=0 .
$$

Similarly, the magnetic scalar potential is expanded in the form

$$
\psi(r, \phi, z)=2 \sin \phi \sum_{n=0}^{\infty} g_{n}(r, z)
$$

where

$$
g_{0}(r, z)=-\frac{1}{r}-\frac{r}{[r(z)]^{2}}
$$

and the other $g_{n}$ are determined by solving the recursion relations

$$
\frac{\partial^{2} g_{n}}{\partial r^{2}}+\frac{1}{r} \frac{\partial g_{n}}{\partial r}-\frac{g_{n}}{r^{2}}=-\frac{\partial^{2} g_{n-1}}{\partial z^{2}}
$$

subject to the boundary conditions

$$
\begin{aligned}
{\left[\frac{\partial g_{n}(r, z)}{\partial r}-r^{\prime}(z) \frac{\partial g_{n-1}(r, z)}{\partial z}\right]_{r=r(z)} } & =0 \quad \text { and } \\
g_{n}(0, z) & =0 .
\end{aligned}
$$

Using MATHEMATICA [8], we have found the first eight terms in the expansion

$$
\operatorname{Im} Z_{\perp}(0)=\frac{Z_{0}}{2 \pi} \sum_{N=1}^{\infty} Z^{(N)} .
$$

Assuming that the boundary derivatives vanish at $z= \pm \infty$ and simplifying the results using partial integration, the first three terms are given by 


$$
\begin{aligned}
Z^{(1)}= & -\int_{-\infty}^{\infty} d z \frac{r^{\prime 2}}{r^{2}}, \\
Z^{(2)}= & \int_{-\infty}^{\infty} d z\left[\frac{1}{2} \frac{r^{\prime 4}}{r^{2}}+\frac{1}{6} r^{\prime \prime 2}\right], \\
Z^{(3)}= & -\int_{-\infty}^{\infty} d z\left[\frac{31}{80} \frac{r^{\prime 6}}{r^{2}}+\frac{45}{64} r^{\prime 2} r^{\prime 2}-\frac{1}{128} r r^{\prime \prime 3}\right. \\
& \left.+\frac{17}{384} r^{2} r^{\prime \prime \prime 2}\right] .
\end{aligned}
$$

Before partial integration each term in $Z^{(N)}$ contains derivatives of $r(z)$ up to order $2 N$. This means that the $N$ th order term $Z^{(N)}$ exists only if the boundary $r(z)$ is smooth enough to have integrable (products of) derivatives up to the $2 N$ th order. The first term $(N=1)$ is Yokoya's approximation. For a piecewise linear tapered structure, the higher-order $(N>1)$ terms require care in their evaluation, because the products of the derivatives are not defined at the corner points. In what follows, we shall treat a linearly tapered structure by considering it to be the limit of a sequence of tapers with smoothed corners. It is interesting to note that all the higher-order terms are proportional to at least one derivative of the radius of the pipe of order two or more. Therefore, for a linearly tapered structure, the corrections to the Yokoya impedance arise due to the change in slope required to match to the entrance and exit chambers.

We have found that, despite partial integration, expressions for $Z^{(N)}$ rapidly lengthen for higher $N$. However, apart from numerical coefficients, the basic structure of the expressions for $Z^{(N)}$ is rather simple. Specifically, when they are ordered in increasing powers of $r(z)$, the first term in the integrand for $Z^{(N)}$ has the form

$$
F^{(N)}=(-)^{N} A_{N} r^{-2} r^{\prime 2 N},
$$

and the last has the form

$$
G^{(N)}=(-)^{N} B_{N}\left(r^{N-2} r^{(N)}\right)^{2},
$$

where $A_{N}$ and $B_{N}$ are positive numerical coefficients, and $G^{(1)} \equiv F^{(1)}$. Intermediate terms include various permutations of powers of $r(z)$ and its derivatives of order $1 \leq m \leq$ $N$ as allowed by dimensionality.

Let us consider a smooth perturbation of the wall radius,

$$
r(z)=r_{a v}[1+\varepsilon b(z / L)]
$$

where $b^{\prime}( \pm \infty)=0$ and $b( \pm \infty)= \pm 1$. Although it is not necessary, we shall assume that $-1 \leq b(z) \leq 1$. In this case,

$$
\begin{aligned}
r_{a v} & =\left(r_{\max }+r_{\min }\right) / 2 \text { and } \\
\varepsilon & =\left(r_{\max }-r_{\min }\right) /\left(r_{\max }+r_{\min }\right) .
\end{aligned}
$$

From inspection of the first three terms given in Eq. (2.11), as well as the next five terms that we do not present in detail, it follows that the perturbation expansion has the form

$$
\operatorname{Im} Z_{\perp}(0)=\frac{Z_{0} \varepsilon^{2}}{2 \pi L} \sum_{N=1}^{\infty}(-)^{N} C_{N}(\varepsilon)\left(r_{a v} / L\right)^{2(N-1)},
$$

where the coefficients $C_{N}(\varepsilon)$ are determined by inserting the radius (2.14) into Eqs. (2.11), for $N \leq 3$, and similar equations not explicitly shown for larger $N$. The coefficients $C_{N}(\varepsilon)$ are positive for the cases we have considered.

\section{B. Smooth structure and small $\varepsilon$}

Let us consider a smooth taper described by Eq. (2.14), with $\varepsilon \ll 1$. In this case, expressions for $Z^{(N)}$ greatly simplify because the "last terms" given by Eq. (2.13) dominate. To $O\left(\varepsilon^{2}\right)$ these terms simplify further because we can replace $r(z)$ by its "average value" $r_{a v}$. For convenience let us measure the pipe radius in units of $r_{a v}$ and axial distance $z$ in units of $L$ - the characteristic distance over which significant tapering occurs. The boundary is now given by

$$
r(z)=\varepsilon b(z)+1,
$$

where $b(z)$ is such that $b(\infty)=-b(-\infty)=1$, and varies mainly in the $|z|<1$ interval.

Formally summing the dominant terms over all perturbation theory orders, we get

$$
\begin{aligned}
\operatorname{Im} Z_{\perp}(0)= & \frac{\varepsilon^{2} Z_{0}}{2 \pi L} \sum_{N=1}^{\infty}\left(\frac{r_{a v}}{L}\right)^{2(N-1)} \\
& \times(-)^{N} B_{N} \int_{-\infty}^{\infty} d z b^{(N)}(z)^{2} .
\end{aligned}
$$

The values of the first eight coefficients $B_{N}$ are given in Table I. The sum in Eq. (2.18) is divergent at large $N$, but it provides a useful asymptotic representation of the impedance. In Appendix A, we show that Eq. (2.18) agrees with the result of the boundary perturbation method $[6,7]$ to $O\left(\varepsilon^{2}\right)$.

For $N>1$, the following representation is found to provide better than $2 \%$ relative accuracy:

$$
B_{N} \cong c_{1} \lambda_{1}^{N}+c_{2} \lambda_{2}^{N},
$$

where $c_{1}=1.67, \lambda_{1}=0.295, c_{2}=5.25, \lambda_{2}=0.0671$. Hence, for large $N, B_{N} \sim c_{1} \lambda_{1}^{N}$, so the product $\left(r_{a v} / L\right)^{2(N-1)} B_{N}$ goes to zero exponentially (if $r_{a v} / L$ is not large). On the other hand, $b^{(N)}$ contains a factorial, and therefore scale as $N^{2 N}$ at large $N$, causing the sum in Eq. (2.18) to be divergent.

TABLE I. Values of the first eight coefficients $B_{N}$.

\begin{tabular}{ccccccccc}
\hline \hline$N$ & 1 & 2 & 3 & 4 & 5 & 6 & 7 & 8 \\
\hline$B_{N}$ & 1 & $\frac{1}{6}$ & $\frac{17}{384}$ & $\frac{49}{3840}$ & $\frac{4141}{1105920}$ & $\frac{17081}{15482880}$ & $\frac{1289549}{3963617280}$ & $\frac{20540423}{214035333120}$ \\
\hline \hline
\end{tabular}


In order to illustrate the behavior of the asymptotic series (2.18), let us consider the smooth boundary given by the error function, $b(z)=\operatorname{Erf}(z / \sqrt{2})$. Computing the derivatives we derive the series

$$
\operatorname{Im} Z_{\perp}(0)=\frac{\varepsilon^{2} Z_{0}}{2 \pi L} \sum_{N=1}^{\infty}(-)^{N} B_{N} \frac{(2 N-3) ! !}{\sqrt{\pi} 2^{N-2}}\left(\frac{r_{a v}}{L}\right)^{2(N-1)} .
$$

In the limit when $r_{a v} / L$ is small,

$$
\operatorname{Im} Z_{\perp}(0) \cong \frac{\varepsilon^{2} Z_{0}}{2 \pi}\left[Z^{(1)}+Z^{(2)}\right],
$$

where $Z^{(1)}$ and $Z^{(2)}$ are the first and the second terms in the sum in Eq. (2.20),

$$
Z^{(1)}=-\frac{2}{\sqrt{\pi} L}, \quad \text { and } \quad Z^{(2)}=\frac{1}{6 \sqrt{\pi}} \frac{r_{a v}^{2}}{L^{3}} .
$$

\section{Linear taper with sharp corners}

Our goal now is to find the impedance of the most basic piecewise linear taper,

$$
r(z)=\left\{\begin{array}{cc}
r_{\min }=r_{a v}(1-\varepsilon) & (z \leq-L) \\
r_{a v}\left(1+\frac{\varepsilon z}{L}\right) & (-L<z<L) \\
r_{\max }=r_{a v}(1+\varepsilon) & (L \leq z)
\end{array}\right\}
$$

As mentioned above, we cannot directly apply Eqs. (2.11) for $N>1$ because at the corner points the second derivative of the boundary is a $\delta$-function, and products of the derivatives of the delta function are not integrable. To treat this problem, we make use of the representation,

$$
\delta(z)=\lim _{a \rightarrow 0} \frac{1}{\sqrt{2 \pi} a} \exp \left(-\frac{z^{2}}{2 a^{2}}\right)
$$

to generate a sequence of smooth boundaries that converge to the linearly tapered structure as $a \rightarrow 0$. We define

$$
\begin{aligned}
b(z)= & \frac{a}{\sqrt{2 \pi}}\left[-\exp \left(-\frac{(z-1)^{2}}{2 a^{2}}\right)+\exp \left(-\frac{(z+1)^{2}}{2 a^{2}}\right)\right] \\
& -\frac{z-1}{2} \operatorname{Erf}\left(\frac{z-1}{\sqrt{2} a}\right)+\frac{z+1}{2} \operatorname{Erf}\left(\frac{z+1}{\sqrt{2} a}\right) .
\end{aligned}
$$

In the limit $a \rightarrow 0$, this function approaches a linear taper with a unit slope in the region $|z|<1$, and $|b(z)|=1$, for $|z| \geq 1$. For small values of $a$ it provides a good approximation to a linear taper. Parameter $a$ determines the characteristic size of the corner region, more specifically it equals the rms spread of the second derivative $b^{\prime \prime}(z)$ near the corners. See Fig. 1.

In case of a shallow taper, $\varepsilon \ll 1$, we can proceed as above, to derive the asymptotic series for the impedance $(a \ll 1)$

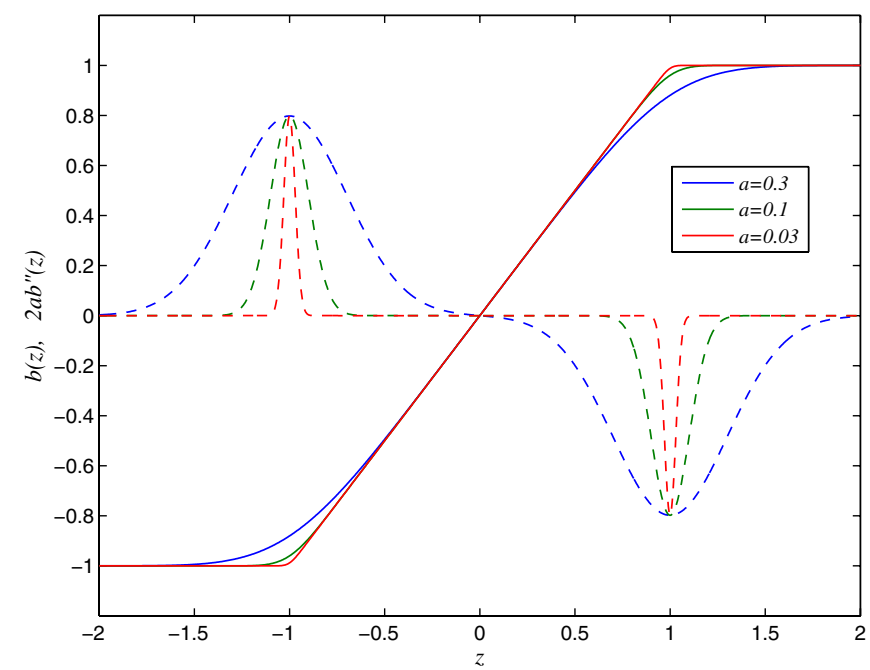

FIG. 1. (Color) $b(z)$ (solid) and $2 a b^{\prime \prime}(z)$ (dash) for $a=0.3,0.1$, and 0.03 .

$$
\begin{aligned}
\operatorname{Im} Z_{\perp}(0) \cong & \frac{\varepsilon^{2} Z_{0}}{2 \pi L}\left[-2+\frac{2 a}{\sqrt{\pi}}+\frac{1}{\sqrt{\pi}} \frac{a^{3} L^{2}}{r_{a v}^{2}}\right. \\
& \left.\times \sum_{N=2}^{\infty}(-)^{N} B_{N} \frac{(2 N-5) ! !}{2^{N-2}}\left(\frac{r_{a v}}{a L}\right)^{2 N}\right]
\end{aligned}
$$

Not surprisingly, this formula is similar to Eq. (2.20) derived for a smooth structure, except inside the sum $L$ gets replaced by the characteristic corner size $a L$. In order to explore the behavior in the limit $a \rightarrow 0$, we shall carry out a Borel summation. First, we replace the factorial in Eq. (2.24) by the integral representation

$(2 N-5) ! !=\frac{2^{N-2}}{\sqrt{\pi}} \Gamma\left(N-\frac{3}{2}\right)=\frac{2^{N-2}}{\sqrt{\pi}} \int_{-\infty}^{\infty} d t t^{N-(5 / 2)} e^{-t}$.

Then we interchange the summation and integration, and use the approximation for $B_{N}$ given in Eq. (2.19). The resulting geometric series can be summed and we obtain

$$
\begin{aligned}
\operatorname{Im} Z_{\perp}(0) \cong & \frac{\varepsilon^{2} Z_{0}}{2 \pi L}\left\{-2+\frac{2 a}{\sqrt{\pi}}+\sum_{j=1}^{2} c_{j} \lambda_{j}^{3 / 2} \frac{r_{a v}}{L}\right. \\
& \left.\times \exp \left(\frac{a^{2} L^{2}}{\lambda_{j} r_{a v}^{2}}\right)\left[1-\operatorname{Erf}\left(\frac{a L}{r_{a v} \sqrt{\lambda_{j}}}\right)\right]\right\}
\end{aligned}
$$

In the limit of sharp corners $(a \rightarrow 0)$, this simplifies to

$$
\begin{aligned}
\operatorname{Im} Z_{\perp}(0) & \cong \frac{\varepsilon^{2} Z_{0}}{2 \pi L}\left(-2+\left(c_{1} \lambda_{1}^{3 / 2}+c_{2} \lambda_{2}^{3 / 2}\right) \frac{r_{a v}}{L}\right) \\
& \cong \frac{\varepsilon^{2} Z_{0}}{2 \pi L}\left(-2+0.36 \frac{r_{a v}}{L}\right) .
\end{aligned}
$$

The first term is that of Yokoya and the second is negligible for small $r_{a v} / L$. In Appendix A, we provide an alternate 
derivation of Eq. (2.28) using the boundary perturbation method [6,7].

Finally, we note that, for the cornered structure, we can drop the requirement for small $\varepsilon$ and basically keep the same derivation. Indeed, comparing various terms in Eqs. (2.11), we have found that the last term $\left(\sim a^{3-2 N}\right)$ dominates for any $\varepsilon$ in the limit of small $a$. Keeping only this term and replacing $r(z)$ by its values $\pm(1-\varepsilon)$ at the corners, we have found that the sum of higher-order terms acquires an extra factor of $\left(1-\varepsilon^{2}\right)^{-1}$. The same factor appears in the Yokoya coefficient $Z^{(1)}$, so we have derived the following result:

$$
\operatorname{Im} Z_{\perp}(0) \cong \frac{\varepsilon^{2} Z_{0}}{\pi L\left(1-\varepsilon^{2}\right)}\left(-1+0.18 \frac{r_{a v}}{L}\right),
$$

as reported in Eq. (1.3) of the Introduction. Equation (2.29) is accurate up to $O[\varepsilon]^{2}$ when $\varepsilon$ is small, however it only partially accounts for the terms of $O[\varepsilon]^{4}$ and higher. The latter could be found to any order, by including additional terms in the expressions for the coefficients $Z^{(N)}$ and employing an analysis similar to the above, which would result in additional terms in parenthesis in Eq. (2.29) of the form $\left(\varepsilon \frac{r_{a v}}{L}\right)^{2},\left(\varepsilon \frac{r_{a v}}{L}\right)^{4}$, etc.

\section{ABCI RESULTS AND COMPARISON TO ANALYTICAL CALCULATIONS}

The discussions presented in Sec. II as well as our previous numerical work [9] have shown that the impedance is conveniently parametrized in terms of $r_{a v} / L$ and $\varepsilon$. To study the dependence of the impedance on these parameters, we have performed extensive calculations for the linear tapered structure shown in Fig. 2. For all of our calculations, we fixed $r_{\min }=1 \mathrm{~cm}$ and $g=20 \mathrm{~cm}$ (after it was checked that increasing $g$ does not alter the results, see Appendix B). The taper lengths $2 L$ and $r_{\max }$ were varied over a broad range. The reasons for using this geometry, as well as the details of the ABCI calculations, are discussed in Appendix B.

$\mathrm{ABCI}$ results for $\varepsilon=1 / 3$ and $17 / 19$ (corresponding to $r_{\max }$ equal to 2 and $18 \mathrm{~cm}$ ) are presented in Fig. 3, together with the estimates from the Yokoya expression (dot-dashed curves),

$$
\operatorname{Im} Z_{\perp}(0) \cong-(2) \frac{Z_{0} \varepsilon}{2 \pi r_{a v}} \frac{2 \tan \theta}{1-\varepsilon^{2}},
$$

doubled due to dual tapered structure. Also shown in Fig. 3 (dashed curves) are the estimates from Eq. (2.29), which we now write in the form

$$
\operatorname{Im} Z_{\perp}(0) \cong-(2) \frac{Z_{0} \varepsilon}{2 \pi r_{a v}} \frac{2 \tan \theta}{1-\varepsilon^{2}}\left(1-0.18 \frac{\tan \theta}{\varepsilon}\right) .
$$

First of all, we see that the Yokoya expression (3.1) is indeed accurate when $r_{a v} / L=\varepsilon^{-1} \tan \theta$ is small. Conversely, while accurate for very gradual and deep struc-

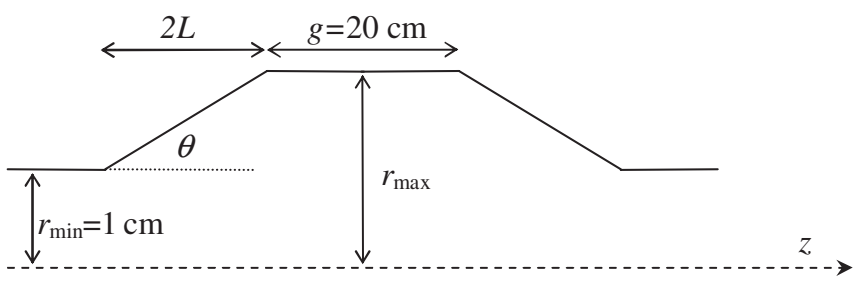

FIG. 2. Geometry used for ABCI calculations. Structure is axially symmetric and mirror symmetric in the $z$ direction.

tures, Yokoya's expression tends to significantly overestimate the impedance when the tapering is not as gradual and/or structures are shallow. In Fig. 3, we also see that Eq. (3.2) extends the agreement with ABCI calculations into the region of steeper tapering.

To proceed, we next replace $\tan \theta$ by $\theta$ in Eq. (3.2) to obtain

$$
\operatorname{Im} Z_{\perp}(0) \cong-(2) \frac{Z_{0} \varepsilon}{2 \pi r_{a v}} \frac{2 \theta}{1-\varepsilon^{2}}\left(1-0.18 \frac{\theta}{\varepsilon}\right) .
$$

We see from Fig. 3 (solid curves) that Eq. (3.3) exhibits better agreement with the $\mathrm{ABCl}$ results for cases of significant change in the cross section $\left(r_{\max } \geq 2 r_{\min }\right.$ or $\left.\varepsilon \geq 1 / 3\right)$. In fact it agrees with $\mathrm{ABCI}$ results with accuracy better than $10 \%$ for tapering angles up to $\pi / 4$, and the agreement extends even further in $\theta$ for deeper structures.

Agreement can be further improved by considering the expression

$$
\operatorname{Im} Z_{\perp}(0) \cong-(2) \frac{Z_{0} \varepsilon}{2 \pi r_{a v}} \frac{2 \theta}{1-\varepsilon^{2}} \frac{1}{1+0.18 \frac{\theta}{\varepsilon}},
$$

which agrees with Eq. (3.3) for $0.18 \theta / \varepsilon \ll 1$. Our final result was obtained by assuming the impedance to be expressed in the form

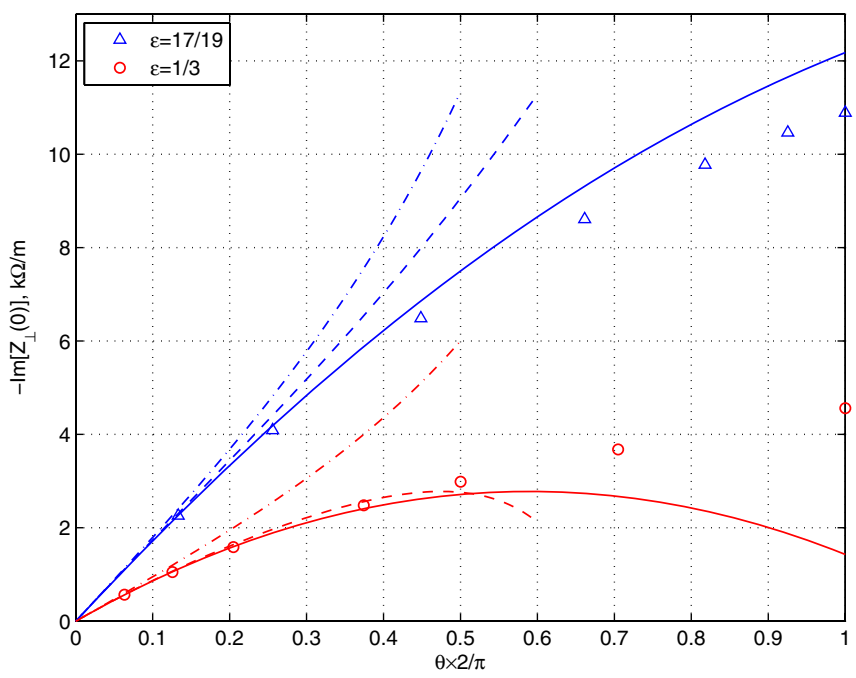

FIG. 3. (Color) Zero-frequency transverse impedance from ABCI (symbols), Yokoya formula Eq. (3.1) (dot-dashed), Eq. (3.2) (dashed), Eq. (3.3) (solid). 


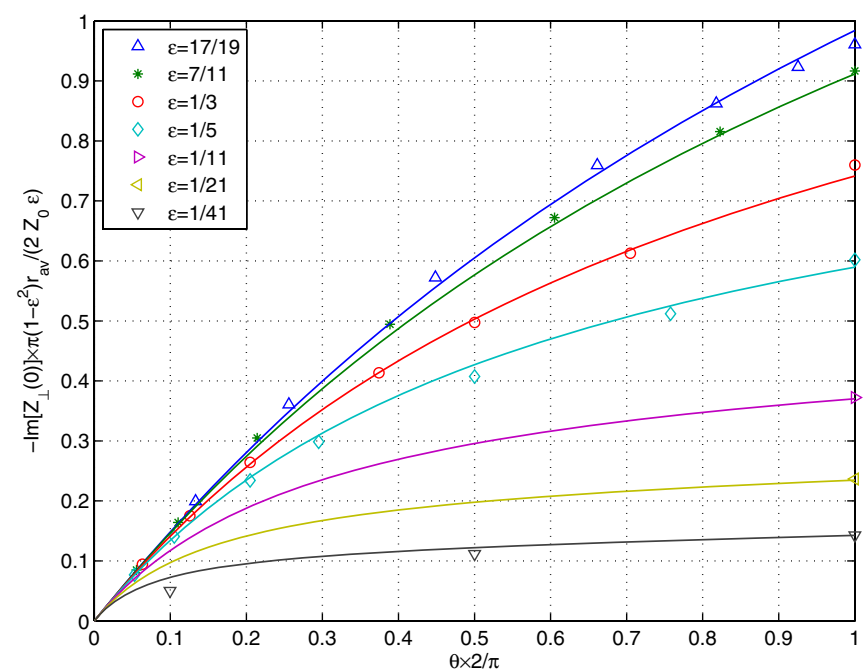

FIG. 4. (Color) The dimensionless scaled impedance as calculated from $\mathrm{ABCI}$ (symbols) and the curves corresponding to approximation given in Eqs. (3.5) and (3.6) (solid).

$$
Z_{\perp}(0) \cong-(2) \frac{i Z_{0} \varepsilon}{2 \pi r_{a v}} \frac{2 \theta}{1-\varepsilon^{2}} \frac{1+(a+b \varepsilon) \frac{\theta}{\varepsilon}}{1+(0.18+a+c \varepsilon) \frac{\theta}{\varepsilon}}
$$

and determining the parameters by carrying out a least squares fit to the ABCI data. In this manner, we found

$$
\begin{gathered}
a=2.94 \times 10^{-3}, \quad b=-3.13 \times 10^{-3}, \\
c=1.75 \times 10^{-1} .
\end{gathered}
$$

In Fig. 4, we plot the ABCI results for the scaled impedance $-\operatorname{Im} Z_{\perp}(0) \pi\left(1-\varepsilon^{2}\right) r_{a v} /\left(2 Z_{0} \varepsilon\right)$ versus the angle $\theta$ in radians divided by $\pi / 2$. The characters as specified in the figure legend are the results of the ABCI calculations and the solid curves show the approximation given by Eqs. (3.5) and (3.6). Note that this excellent fit over all angles $0 \leq \theta \leq \pi / 2$ and most of the range $0<\varepsilon<1$ is obtained using only three free parameters.

\section{CONCLUDING REMARKS}

We have considered the transverse impedance of a tapered structure in axially symmetric geometry. The main results of our paper can be summarized as follows:

(i) We extended the perturbation theory method of [3] to higher orders.

(ii) This extension allowed us to identify the small parameter $r_{a v} / L$. It is only when this parameter is small that the Yokoya expression Eq. (1.1) is valid.

(iii) Summing higher orders of the perturbation theory we obtained the analytical expression Eq. (1.5) for the impedance of a linear taper with corners included, extending much further in tapering angle than Eq. (1.1). (iv) We performed extensive calculations with $\mathrm{ABCI}$ for linearly tapered structures, confirming the analytical results above.

(v) Combining our analytical and numerical results, we have developed an empirical parametrization Eqs. (1.7) and (1.8) that well approximates all of our ABCI results, spanning the relevant parameter regimes for most applications.

(vi) Future applications of this work include extension to structures of elliptical and flat cross sections [5], as well as impedance minimization by means of nonlinear tapering.

\section{ACKNOWLEDGMENTS}

This work was supported by Department of Energy Contract No. DE-AC02-98CH10886.

\section{APPENDIX A: RESULTS OF BOUNDARY PERTURBATION METHOD}

Let us consider a smooth perturbation of the wall radius,

$$
r(z)=r_{a v}[1+\varepsilon S(z)]
$$

where

$$
S(z)=\int_{-\infty}^{\infty} d q A(q) e^{-i q z}
$$

A perturbation expansion in powers of $\varepsilon$ has been developed [6] and to leading order [2]

$$
\begin{aligned}
\operatorname{Im} Z_{\perp}(0)= & -2 Z_{0} \varepsilon^{2} \int_{-\infty}^{\infty} d q q^{2}|A(q)|^{2}\left(\frac{I_{1}{ }^{\prime}\left(q r_{a v}\right)}{q r_{a v} I_{1}\left(q r_{a v}\right)}\right. \\
& \left.-\frac{I_{1}\left(q r_{a v}\right)}{\left(q r_{a v}\right)^{3} I_{1}{ }^{\prime}\left(q r_{a v}\right)}\right) .
\end{aligned}
$$

Using MATHEMATICA, it is easy to show that

$$
-2\left(\frac{I_{1}{ }^{\prime}(x)}{x I_{1}(x)}-\frac{I_{1}(x)}{x^{3} I_{1}{ }^{\prime}(x)}\right)=\sum_{N=1}^{\infty}(-)^{N} B_{N} x^{2(N-1)},
$$

where the coefficients $B_{N}$ are those introduced in Eq. (2.13), see Table I. Now let us assume

$$
S(z)=b(z / L) \text {. }
$$

Inserting Eq. (A4) into Eq. (A3), and using the identity

$$
\int_{-\infty}^{\infty} d z S^{(N)}(z)^{2}=2 \pi \int_{-\infty}^{\infty} d q q^{2 N}|A(q)|^{2},
$$

we obtain the asymptotic series

$$
\begin{aligned}
\operatorname{Im} Z_{\perp}(0)= & \frac{\varepsilon^{2} Z_{0}}{2 \pi L} \sum_{N=1}^{\infty}\left(\frac{r_{a v}}{L}\right)^{2(N-1)} \\
& \times(-)^{N} B_{N} \int_{-\infty}^{\infty} d z b^{(N)}(z)^{2},
\end{aligned}
$$

in agreement with Eq. (2.18) derived using the extension of Stupakov's perturbation theory. 
It has been shown [7] that Eq. (A3) can be rewritten in the form

$$
\operatorname{Im} Z_{\perp}(0)=\frac{-Z_{0} \varepsilon^{2}}{\pi r_{a v}^{2}} \sum_{n=1}^{\infty}\left\{\frac{F\left(k_{n}{ }^{\prime}\right)}{\left(\nu_{n}^{2}-1\right) k_{n}{ }^{\prime}}+\frac{F\left(k_{n}\right)}{k_{n}}\right\},
$$

where

$$
\begin{gathered}
k_{n}=\frac{\mu_{n}}{r_{a v}}, \quad k_{n}{ }^{\prime}=\frac{\nu_{n}}{r_{a v}}, \\
J_{1}\left(\mu_{n}\right)=0, \quad J_{1}{ }^{\prime}\left(\nu_{n}\right)=0,
\end{gathered}
$$

and

$$
F(p)=2 \int_{-\infty}^{\infty} d z_{1} \int_{-\infty}^{z_{1}} d z_{2} S^{\prime}\left(z_{1}\right) S^{\prime}\left(z_{2}\right) e^{-p\left(z_{1}-z_{2}\right)}
$$

For a linear taper, we take

$$
S^{\prime}(z)=\left\{\begin{array}{cc}
1 / L & -L<z<L \\
0 & \text { otherwise }
\end{array}\right\}
$$

In this case, we find

$$
\begin{aligned}
\operatorname{Im} Z_{\perp}(0)= & -\frac{2 Z_{0} \varepsilon^{2}}{\pi L} \frac{r_{a v}}{L} \sum_{n=1}^{\infty}\left[\frac{2 \nu_{n} \frac{L}{r_{a v}}-1+e^{-2 \nu_{n} L / r_{a v}}}{\left(\nu_{n}^{2}-1\right) \nu_{n}^{3}}\right. \\
& \left.+\frac{2 \mu_{n} \frac{L}{r_{a v}}-1+e^{-2 \mu_{n} L / r_{a v}}}{\mu_{n}^{3}}\right] .
\end{aligned}
$$

When $L / r_{a v} \gg 1$, this expression simplifies and we obtain

$$
\operatorname{Im} Z_{\perp}(0) \cong-\frac{2 Z_{0} \varepsilon^{2}}{\pi L}\left(C_{0}-C_{1} \frac{r_{a v}}{L}\right)
$$

with

$$
\begin{gathered}
C_{0}=2 \sum_{n=1}^{\infty}\left[\frac{1}{\left(\nu_{n}^{2}-1\right) \nu_{n}^{2}}+\frac{1}{\mu_{n}^{2}}\right]=\frac{1}{2}, \\
C_{1}=\sum_{n=1}^{\infty}\left[\frac{1}{\left(\nu_{n}^{2}-1\right) \nu_{n}^{3}}+\frac{1}{\mu_{n}^{3}}\right]=0.900 .
\end{gathered}
$$

In this manner we confirm the result derived earlier in Eq. (2.28):

$$
\operatorname{Im} Z_{\perp}(0)=-\frac{Z_{0} \varepsilon^{2}}{\pi L}\left(1-0.18 \frac{r_{a v}}{L}\right)
$$

\section{APPENDIX B: DETAILS OF ABCI CALCULATIONS}

\section{Preliminaries}

For our ABCI calculations we have chosen a basic piecewise linear tapered structure shown in Fig. 2. As ABCI requires equal cross sections of the input and output pipes [4], we utilized a dual taper. Since we are studying the impedance of a taper (rather than possible interference between two tapers) we have chosen the length of the middle section $g$ to be long enough to assure that its lengthening does not affect the results. In other words, this geometry is suitable for studying the regime of two separate steps. Also, we have chosen long enough outer pipes to not affect our results. Our initial calculations [9] were performed for a concave structure, i.e., with an outer pipe radius larger than that of the midsection. For the present paper we ended up switching to a convex structure (Fig. 2), which (due to some unimportant systematic effects of $\mathrm{ABCI}$ code) has generally allowed us to use a more coarse mesh. However, we did confirm that in the limit of vanishing mesh size the results for the zero-frequency impedance for convex and concave configurations are essentially the same, which is consistent with the physical intuition.

\section{Calculating zero-frequency impedance}

Being a time domain code, $\mathrm{ABCI}$ does not find the impedance directly but rather computes the values of the wake potential (in units of $\mathrm{V} / \mathrm{pC} / \mathrm{m}$ ) on a mesh extending some distance $s_{\max }$ behind a Gaussian bunch of rms length $\sigma_{z}$. While the wake-potential and the zero-frequency transverse impedance are simply related by

$$
Z_{\perp}(0)=\frac{1}{c} \int_{-\infty}^{\infty} d z W_{\perp}^{\sigma_{z}}(z)
$$

it took some effort to develop a reliable algorithm for choosing the mesh size, rms bunch length $\sigma_{z}$, and integration limit $s_{\max }$ such that the quantity we extract from the ABCI using

$$
Z_{\perp}(0)=\frac{10^{12}}{c} \int_{-5 \sigma_{z}}^{s_{\max }} d z W_{\perp}^{\mathrm{ABCI}}(z)
$$

accurately represented the low frequency impedance of the tapered structures under consideration. ${ }^{1}$

One of our consistency checks is illustrated in Fig. 5 where we plot $\mathrm{ABCI}$ wake potentials for a fairly gradual tapered structure. The interaction of a bunch having $\sigma_{z}$ in $\mathrm{cm}$-range with this structure is expected to be well within the Yokoya regime. It is seen that apart from the shortest bunch length, $\sigma_{z}=0.3 \mathrm{~cm}$, the wake potentials pretty well line up with the driving bunch and are predominantly of Gaussian shape, i.e., they correspond to purely inductive impedance. Furthermore, while the length of the drive bunch varies by more than an order of magnitude, scaled wake potentials overlay quite well on top of each other. In fact, the areas under the curves in Fig. 5 are within a

\footnotetext{
${ }^{1}$ While both integration limits are variable, usually there is no need to adjust the lower one. As for $s_{\max }$ we usually set it so that the oscillations in the long range wake are down to a fraction of a percent of its maximum value. We used a trapezoidal method for the integral in Eq. (B2). One obtains the same value for $Z_{\perp}(0)$ using built-in Fourier transform in ABCI provided data windowing option is disabled.
} 


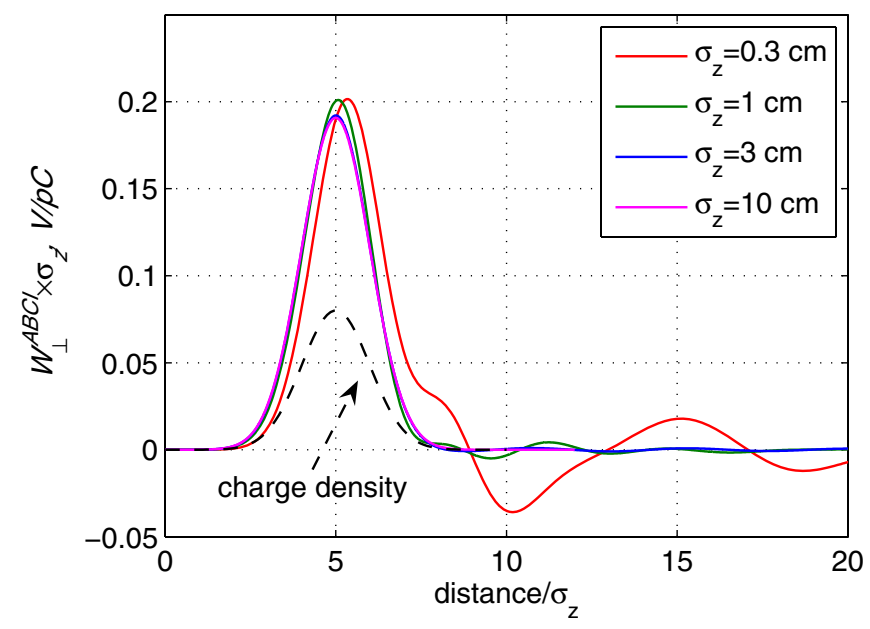

FIG. 5. (Color) Scaled wake potentials from ABCI for varying rms bunch length. Geometry is that of Fig. 2, with $\varepsilon=7 / 11$ and $r_{a v} / L=0.275(\theta=173 \mathrm{mrad})$; mesh size is $100 \mu \mathrm{m}$.

fraction of a percent of each other, ${ }^{2}$ indicating constant impedance vs frequency.

Therefore our procedure is consistent at least in the range of $\sigma_{z}$ considered. For calculations in this paper, we used rms bunch length of $1-10 \mathrm{~cm}$ and for many structures the results were confirmed for several values of $\sigma_{z}$.

\section{Mesh size}

It is well known that calculations of gradually tapered structures require very fine mesh. Some considerations on the choice of mesh size specific to ABCI code are presented in [4]; however they do not directly apply to the low frequency regime we consider. Newer codes have been written in part to specifically reduce the need for extremely fine mesh $[10,11]$. We believe that we found a reliable algorithm for using $\mathrm{ABCI}$ to determine the zero-frequency impedance without resorting to extremely fine mesh, which is described below.

For every $\mathrm{ABCI}$ run, we fixed the radial and longitudinal mesh sizes to be equal to each other and made sure that the principle dimensions of the structure $\left(r_{\min }, r_{\max }, 2 L\right)$ divide evenly into this mesh size. Refining the mesh we observed linear decrease of impedance as defined by Eq. (B2) that continued down to the finest mesh we could afford. For one particular geometry, this is illustrated in Fig. 6. The magnitude of the slope did vary with geometry, specifically a larger slope occurred for more gradual tapers, however the linear decrease with mesh size was observed for every structure calculated. On top of that, for several geometries we have independently confirmed this behavior with a 3D time domain code GDFIDL [12], which has shown very good

\footnotetext{
${ }^{2}$ This does include the $\sigma_{z}=0.3 \mathrm{~cm}$ curve, which however had to be integrated to $s_{\max }=67 \sigma_{z}$ where ringing becomes negligible.
}

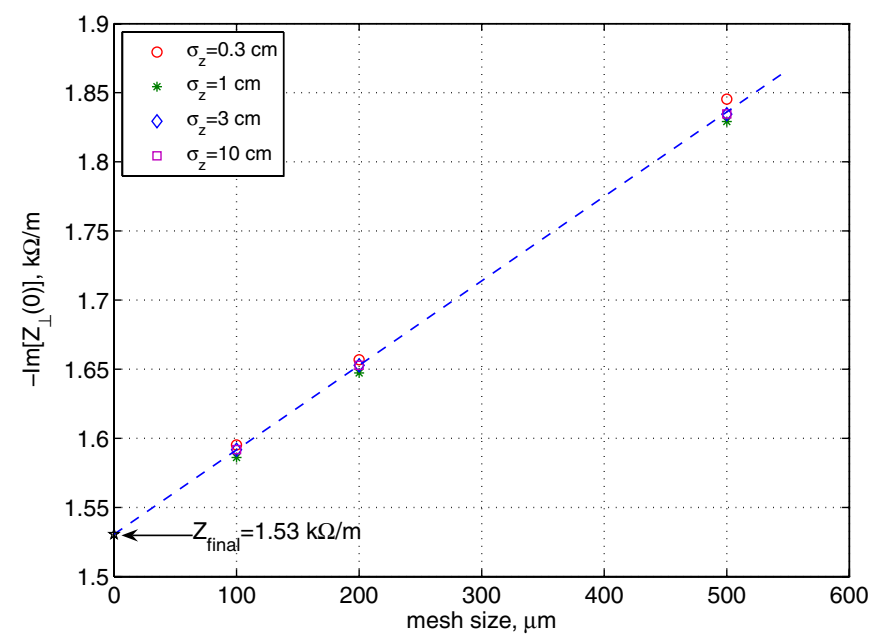

FIG. 6. (Color) Impedance calculated from ABCI using Eq. (B2) (color symbols), and linear interpolation to zero mesh size (dash). Geometry is that of Fig. 2, with $\varepsilon=7 / 11$ and $r_{a v} / L=$ $0.275(\theta=173 \mathrm{mrad})$.

agreement with $\mathrm{ABCI}$. We believe that this linear decrease is physically meaningful, and that its extrapolation to zero mesh gives a good estimate of zero-frequency impedance.

In order to take full advantage of this observation, we adopted the following approach. Depending on the overall size and the taper angle of the structure, the initial mesh size was chosen in the range of $200-500 \mu \mathrm{m}$. It was subsequently refined by a factor of 2 , until the $\mathrm{ABCI}$ results given by Eq. (B2) for two consecutive mesh sizes agreed to $5 \%$ or better. Once this condition was satisfied, we linearly interpolated the two latest results to zero mesh size, which was then taken as the final result for each structure $\left(Z_{\text {final }}\right.$ in Fig. 6) and reported through the rest of the paper.

[1] A. W. Chao, Physics of Collective Beam Instabilities in High Energy Accelerators (Wiley, New York, 1993).

[2] K. Yokoya, CERN SL/90-88 (AP), 1990.

[3] G. V. Stupakov, SLAC-PUB-7086, 1995.

[4] Y.-H. Chin, CERN SL/94-02 (AP), 1994.

[5] B. Podobedov and S. Krinsky (unpublished).

[6] R. K. Cooper, S. Krinsky, and P. L. Morton, Part. Accel. 12, 1 (1982).

[7] S. Krinsky, Phys. Rev. ST Accel. Beams 8, 124403 (2005).

[8] Wolfram Research, http://www.wolfram.com.

[9] B. Podobedov and S. Krinsky, in Proceedings of the 2005 Part. Accelerator Conference, Knoxville, Tennessee (IEEE, Piscataway, NJ, 2005).

[10] A. Novokhatski, M. Timm, and T. Weiland, Proceedings of ICAP '98, Monterey, California, 1998, www.slac.stanford.edu/xorg/icap98.

[11] I. Zagorodnov, T. Weiland, and K. Bane, in Proceedings of the 2003 Particle Accelerator Conference, Oregon (IEEE, Piscataway, NJ, 2003).

[12] W. Bruns, http://www.GdfidL.de. 